\title{
Efficacy of Methotrexate in Combination with Antioxidant Vitamins (A, C and E) versus Methotrexate alone in the Treatment of Rheumatoid Arthritis
}

Kamrul Hasan Lohani ${ }^{1}$, Ratan Kumar Nath ${ }^{1}$, Mahtab Uddin Hasan $^{2}$, Sujat Paul ${ }^{1}$, Mohammed Habibur Rahman ${ }^{1}$, Golam Faruk ${ }^{1}$, Mohammad Nezam Uddin ${ }^{3}$, Rajat Sanker Roy Biswas ${ }^{4}$, Mohammed Rezaul Karim ${ }^{5}$, Mizanur Rahman*6

${ }^{1}$ Chittagong Medical College, Chittagong, Bangladesh

${ }^{2}$ Marine City Medical College, Chittagong, Bangladesh

${ }^{3}$ Treatment Hospital Nasirabad, Chittagong, Bangladesh

${ }^{4}$ Chattagram Maa O Shishu Hospital Medical College, Chittagong,

Bangladesh

${ }^{5}$ Bilaichary Upazilla Health Complex, Rangamati, Bangladesh

${ }^{6}$ Rangamati Medical College, Rangamati, Bangladesh

Citation: Lohani K. H., Nath R. K., Hasan M.U., Paul S., Rahman M.H., Faruk G., Uddin M.N., Biswas R.S.R., Karim M.R., Rahman M. (2018) Efficancy of Methotrexate in Combination with Antioxidant Vitamins ( $A, C$ and $E$ ) versus Methotrexane alone in the Treatment of Rheumatic Arthritis. Open Science Journal 3(1)

Received: $26^{\text {nd }}$ October 2017

Accepted: $28^{\text {th }}$ November 2017

Published: $2^{\text {nd }}$ January 2018

Copyright:@ 2018 This is an open access article under the terms of the Creative Commons Attribution License, which permits unrestricted use, distribution, and reproduction in any medium, provided the original author and source are credited.

Funding: The author(s) received no specific funding for this work

Competing Interests: The author have declared that no competing interests exists.
*Corresponding author: Mizanur Rahman: mizan2011bio@gmail.com

\section{Abstract:}

Background: There is evidence that oxidative stress plays an important role in auto- immune diseases, such as rheumatoid arthritis (RA). Despite the supporting evidence for a beneficial effect of antioxidants on clinical characteristics of RA, the right balance for optimal effectiveness of antioxidants is largely unknown. A study was designed to determine the potential beneficial effects of antioxidant intervention on clinical parameters of RA.

Methods: Randomized clinical trial of 152 patients with positive rheumatoid factor $(\mathrm{RF})$ and a Disease Activity Score (DAS 28) higher than 3.2 were enrolled in the study. Patients were divided into two groups (Group A and Group B) randomly and group A received methotrexate and antioxidant vitamins ( $\mathrm{A}, \mathrm{C}$ and $\mathrm{E}$ in a fixed dose) and group $\mathrm{B}$ methotrexate only. They were followed up for three visits (baseline, $10^{\text {th }}$ week and $14^{\text {th }}$ week). The intervention was stopped after 10 weeks and was followed by a 'wash-out' period of 4 weeks. At baseline, $10^{\text {th }}$ week and $14^{\text {th }}$ week patient's condition were assessed by means of DAS-28 score. P- Value less than $<0.05$ was considered significant. 
Results: The numbers of swollen and tender joints were significantly reduced and general health was improved reflected by improved DAS28 score at $10^{\text {th }}$ week.

The antioxidant effect was considered beneficial as compared to the scores of $1^{\text {st }}$ visit at base line; the DAS-28 score was significantly reduced at $2^{\text {nd }}$ visit at $10^{\text {th }}$ week. Increment of the DAS-28 score among the group A patients who were on antioxidant up to10 weeks, after the "wash-out period" of four weeks i.e. at $14^{\text {th }}$ week confirmed a significant relation between changes in clinical condition and antioxidants.

Conclusion: This study was designed to assess the potential beneficial effect of antioxidants (Vitamin A, C and E) in combination with methotrexate in the treatment of RA.

Keywords: Rheumatoid arthritis, Disease Activity Score (DAS), Methotrexate, Antioxidants

\section{Introduction}

Rheumatoid arthritis (RA) is a chronic, inflammatory disorder that may affect many tissues and organs, but principally attacks flexible synovial joints. The process involves an inflammatory response of the capsule around the joints synovium secondary to swelling (hyperplasia) of synovial cells, excess synovial fluid, and the development of fibrous tissue (pannus) in the synovium. The pathology of the disease process often leads to the destruction of articular cartilage and ankylosis (fusion) of the joints. RA can also produce diffuse inflammation in the lungs, membrane around the heart (pericardium), the membranes of the lung (pleura), and white of the eye (sclera), and also nodular lesions, most common in subcutaneous tissue. Although the cause of RA is unknown, autoimmunity plays a pivotal role in both its chronicity and progression, and RA is considered a systemic autoimmune disease. The diagnosis of RA, particularly early in the course of disease is empirical and imprecise. Treatment of RA may be efficient if starts early. At the same time an early and accurate diagnosis may protect the patients who do not have RA, from aggressive therapies with potential toxicity [1].

About $1 \%$ of the world's population is affected by RA, women three times more often than men. Onset is most frequent between the ages of 40 and 50, but people of any age can be affected. In addition, individuals with the HLA-DR1 or HLA-DR4 serotypes have an increased risk for developing the disorder. It can be a disabling and painful condition, which can lead to substantial loss of functioning and mobility if not adequately treated. It is a clinical diagnosis made on the basis of symptoms, physical examination, radiographs (X-rays) and laboratory findings, although the American College of Rheumatology (ACR) and the European League against Rheumatism (EULAR) published classification criteria for the purpose of research. Diagnosis and long-term management are 
typically performed by a rheumatologist, an expert in joint, muscle and bone diseases [1].

The main aim of management of RA is to:

- $\quad$ beneficially modify the disease process

- $\quad$ educate the patient

- control pain

- optimize function

These aims are interrelated and success in one area often benefits the others. Successful management inevitably requires careful assessment of the person as well as his or her musculo-skeletal system. The management plan needs to be individualized according to:

- the person's daily activity requirements and recreational aspirations

- the person's perceptions and knowledge of his/ her condition

- medications and coping strategies already tried by the patient

- co-morbid disease and it's therapy

- $\quad$ risk factors and associations of the musculoskeletal conditions

The wide variety of treatment approaches may require the expertise of a number of health professionals, necessitating a coordinated multidisciplinary team approach for some patients [2]. Pharmacotherapy for RA generally involves NSAIDs for control of pain, with selective use of low-dose oral or intra-articular glucocorticoids, and initiation of a DMARD (Disease Modifying Anti-Rheumatic Drugs) [3]. In past decades, pharmacologic treatment of RA was managed using a pyramid approach, symptoms alleviating treatment were started at diagnosis, and only with progression of symptoms dosages were changed or additional medications added. However, a "reverse pyramid" approach now is favored, in which DMARDs are initiated quickly to slow disease progression as early as possible. This change of approach is a result of several research findings: (1) joint damage begins early in the disease; (2) DMARDs have significant benefits when used early; (3) the benefits of DMARDs may be enhanced when the drugs are used in combination (4) a number of new DMARDs are available, with good evidence of beneficial effect. Patients with mild disease and normal radiographic findings can begin treatment with hydroxychloroquine, sulfasalazine or minocycline, although methotrexate also is an option. Patients with more severe disease or radiographic changes should begin treatment with methotrexate. If symptoms are not adequately controlled, leflunomide, azathioprine or combination therapy (methotrexate plus one of the newer agents) may be considered.

Antioxidant supplements and diets have long been advocated for the treatment of RA, osteoarthritis (OA) and other inflammatory arthritis [4]. However, the value of antioxidants in the prevention and treatment of a wide range of serious diseases including stroke, cancer, diabetes, cataracts, Parkinson's disease, Alzheimer's disease and arthritis has been questioned in the light of more recent negative research findings and studies suggesting that antioxidant properties may be absent or reduced in vivo, may only be important in those with a deficiency [4]. It therefore seems timely to assess the clinical evidence supporting the use of antioxidants specifically in arthritis. If shown to be safe and 
effective, antioxidants may be an alternative to treatment with non-steroidal anti-inflammatory drugs (NSAIDs) or other drugs that are associated with adverse effects during long-term use.

In $\mathrm{RA}$, reactive oxygen species and other free radicals are associated with the inflammatory process via numerous pathways [5]. These include the role of nitrous oxide in regulating vascular tone, superoxide in fibroblast proliferation and hydrogen peroxide in the transcription of cytokines IL-2 and TNF- $\alpha$. During inflammation, oxidation modifies low-density lipoproteins, inactivates $\alpha-1$ protease inhibitor, damages DNA and causes lipid peroxidation [5]. Reactive oxygen species also damage cartilage and the extracellular matrix and inhibit collagen and proteoglycan synthesis [6]. Evidence that increased oxidative stress or deficient antioxidant status are important in the pathogenesis of RA comes from several studies. Epidemiological studies have shown that low intake of dietary antioxidants is associated with the incidence of RA [4]. Furthermore, animal studies have demonstrated an anti-inflammatory role for some antioxidants including superoxide dismutase (SOD) and vitamin $\mathrm{E}$ in experimentally induced arthritis. Numerous clinical studies testing the effectiveness of specific antioxidants or particular antioxidant diets in the treatment of arthritis have been published during the last 30 yrs. Here, the study was designed for evaluation the effectiveness of the antioxidant vitamins $\mathrm{A}, \mathrm{C}$ and $\mathrm{E}$ and methotrexate in comparison with methotrexate alone in the treatment of RA.

\section{Materials and methods}

A randomized open clinical trial held on department of Medicine, Chittagong Medical College Hospital, Chittagong, Bangladesh from July 2012 to January 2014. All patients of both sexes between 16-70 years who were diagnosed as RA (ARA 2010) were enrolled in this study. Inclusion criteria are diagnosed case of RA by ACR/EULAR (2010) Classification Criteria, age at entry 16-70 years and DAS- $28^{*}$ score $>3.2$ (i.e. moderate to very active diseases). Exclusion Criteria were patient having any arthritis other than RA, patients having contraindication to use methotrexate and antioxidant drugs (e.g. liver disease, renal disease, active tuberculosis and neoplastic diseases, etc), patients receiving antioxidants (Vitamin-A, C \& E) more than three months before the study, patients on steroid therapy for more than three months before the study, pregnant and lactating women.

Classification criteria of RA 7

Score

(2010 ACR-EULAR criteria)

ACR: American College of Rheumatology, EULAR: European League against Rheumatism

\begin{tabular}{||c||c|}
\hline 1 large joint (shoulder, elbow, hip, knee, ankle) & 0 \\
\hline \hline $2-10$ large joints & 1 \\
\hline \hline $1-3$ small joints (MCP, PIP, Thumb IP, MTP, wrists) & 2 \\
\hline
\end{tabular}


Classification criteria of RA 7

(2010 ACR-EULAR criteria)

ACR: American College of Rheumatology, EULAR: European League against Rheumatism

\begin{tabular}{|c|c|c|}
\hline \multirow[b]{2}{*}{ Joint involvement } & $4-10$ small joints & 3 \\
\hline & $>10$ joints (at least 1 small joint) & 5 \\
\hline \multirow{3}{*}{ Serology } & Negative RF and negative ACPA & 0 \\
\hline & Low-positive RF or low-positive anti-CCP antibodies ( $<3$ times ULN) & 2 \\
\hline & High-positive RF or high-positive anti-CCP antibodies ( $>3$ times ULN) & 3 \\
\hline \multirow[t]{2}{*}{ Acute-phase reactants } & Normal CRP and normal ESR & 0 \\
\hline & Abnormal CRP or abnormal ESR & 1 \\
\hline \multirow[t]{2}{*}{ Duration of symptoms } & $<6$ weeks & 0 \\
\hline & $>6$ weeks & 1 \\
\hline
\end{tabular}

The progression of RA can be followed using scores such as DAS-28. The joints included in DAS-28 score were (bilaterally): proximal interphalangeal joints (10 joints), meta- carpophalangeal joints (10), wrists (2), elbows (2), shoulders (2) and knees (2). When looking at these joints, both the number of joints with tenderness upon touching (TEN-28) and swelling (SW-28) were counted.

In addition, the ESR was measured. Also, the patient makes a subjective assessment (SA) of disease activity during the preceding 7 days on a scale between 0 and 100, where 0 was "no activity" and 100 was "highest activity possible". With these parameters, DAS 28 score was calculated as:

$$
D A S 28=0.56 \times \sqrt{T E N 28}+0.28 \times \sqrt{S W 28}+0.70 \times \ln (E S R)+0.014 \times S A
$$

From this, the disease activity of the patient can be classified as follows [5]:

\begin{tabular}{|c|c|c|c|c|}
\hline & $\begin{array}{c}\text { Current DAS 28 } \\
\text { score }\end{array}$ & $\mathbf{3 . 2}$ & $>\mathbf{3 . 2}$ but $\leq \mathbf{5 . 1}$ & $>\mathbf{5 . 1}$ \\
\hline \multirow{3}{*}{$\begin{array}{c}\text { DAS 28 score } \\
\text { decreases from } \\
\text { initial value }\end{array}$} & $>1.2$ & Inactive & Moderate & Very active \\
\cline { 2 - 5 } & $>0.6$ but $\leq 1.2$ & $\begin{array}{c}\text { Mood improvement } \\
\text { improvement }\end{array}$ & $\begin{array}{c}\text { Moderate } \\
\text { improvement }\end{array}$ & $\begin{array}{c}\text { Moderate } \\
\text { improvement }\end{array}$ \\
\cline { 2 - 5 } & \multicolumn{2}{|c|}{$\leq 0.6$} & No improvement & No improvement \\
\hline
\end{tabular}




\section{Procedure of the study}

Total 152 patients of RA were selected for the study. All patients were informed about the treatment options. Written informed consent was taken from the patients. Ethical permission was also obtained from ethical review committee. Among the 152 patients 124 were finally selected who have DAS-28 score more than 3.2 (i.e. moderate to very active disease). Patients were divided in two groups $(\mathrm{A} \& \mathrm{~B})$ according to lottery and documentation of Case Record Form were done. Group A patients were given methotrexate (Batch no. 72E97044, Mfg Date. Aug 2011, Delta Pharmaceuticals Co.) along with Tab Vitamin C (Batch No.6H03256, Mfg Date. Apr 2011, Square Pharmaceuticals Co.) 250mg daily, Tab Vitamin E (Batch No. 2916, Mfg Date. Jul 2011, Renata Pharmaceuticals Co.) 200 IU daily and Cap Vitamin A (Batch No.0619, Mfg Date. Feb 2011, Drug International Ltd.) 50000 IU twice weekly [11] and Group-B patients were given Tab. Methotrexate (Batch No.72E97044, Mfg Date. Aug 2011, Delta Pharmaceuticals Co.) $7.5 \mathrm{mg} / \mathrm{kg}$ body weight/week to $25 \mathrm{mg} / \mathrm{kg}$ body weight/week. All patients were given NSAIDS in the form of Naproxen $500 \mathrm{mg}$ (Batch No.7703, Mfg Date. Mar 2011, Radiant Pharmaceuticals Co.) 12 hourly for 4 weeks and steroid in the form of Prednisolone $1 \mathrm{mg} / \mathrm{Kg}$ body weight for one month and then tapered. Then both groups were observed for the duration, frequency and severity of join pain, morning stiffness. Side effects of the drugs like flu like illness, vomiting, diarrhea and constipation were recorded. Investigations like complete blood count, CRP, SGPT, Serum Creatitine, X-ray hand, RA factor with titre, Anti CCP were done. DAS 28 score was calculated by net calculator (DAS-28 has sensitivity of $87 \%$ and specificity of $67 \%$ ). Base line findings were recorded at $1^{\text {st }}$ visit (0 week), after giving the drugs different findings were recorded at $2^{\text {nd }}$ visit $\left(10^{\text {th }}\right.$ week $)$. Antioxidants were stopped after 10 weeks. Then the findings were again recorded at $3^{\text {rd }}$ visit $\left(14^{\text {th }}\right.$ week). Last 4 weeks were the observation period without antioxidants. At the $10^{\text {th }}$ week 7 patients from Group A and 8 patients from Group B and at the $14^{\text {th }}$ week 7 patients of Group A and 6 patients of Group B were dropped out.

\section{Data Processing and Analysis}

Data cleaning were done and data were entered into computer. Statistical analysis of the results being obtained by using windows based computer software device with SPSS -18(SPSS Inc, Chicago, IL, USA). All data were tested by using statistical methods-Chi-square test, t-test and ANOVA. Qualitative variables like age group, sex were analyzed by Chi-square test and quantitative variables like DAS 28, disease activities like changes in joint pain, swelling and tenderness, duration of morning stiffness, VAS score were analyzed by t-test and ANOVA. The statistical terms included in this study are mean, standard deviation and percentage. Statistical significance is set at $\mathrm{p}<0.05$ and confidence interval set at $95 \%$ level. 


\section{Results}

Table I: Distribution of the age groups among the study groups $(\mathrm{n}=124)$ :

\begin{tabular}{|l|c|c|c|}
\hline Age & $\begin{array}{c}\text { Group A } \\
(\mathrm{n}=63)(\%)\end{array}$ & $\begin{array}{c}\text { Group B } \\
(\mathrm{n}=61)(\%)\end{array}$ & $\begin{array}{c}\text { Total } \\
(\mathrm{n}=124)(\%)\end{array}$ \\
\hline$\leq 30$ & $17(13.7 \%)$ & $16(12.9 \%)$ & $33(26.6 \%)$ \\
\hline $30-40$ & $24(19.4 \%)$ & $10(8.1 \%)$ & $34(27.4 \%)$ \\
\hline $40-50$ & $9(7.3 \%)$ & $2(1.6 \%)$ & $8(17.7 \%)$ \\
\hline $60-60$ & $6(4.8 \%)$ & $2(1.6 \%)$ & $9(7.3 \%)$ \\
\hline Total & $7(5.6 \%)$ & $61(49.2 \%)$ & $124(100.0 \%)$ \\
\hline \multicolumn{2}{|c|}{$63(50.8 \%)$} & \\
\hline \multicolumn{2}{|c|}{$\mathrm{X}$ value $=2.07$ df $=4 . \mathrm{P}=0.08$. Not significant $(\mathrm{P}>0.05)$} \\
\hline
\end{tabular}

In group A, majority of patients were within 30-40 years $(24,19.4 \%)$, and in group B majority were within 40-50 years $(31,25.0 \%)$. Mean ( \pm SD) age was $41.65 \pm 11.04$ years the age difference between two groups were not significant $(\mathrm{p}>0.05)$.

Table II: Clinical manifestation among the study groups $(\mathrm{n}=124)$ :

\begin{tabular}{|c|c|c|}
\hline Features & Group A $(n=63)$ & Group B $(n=61)$ \\
\hline Morning stiffness $>60 \mathrm{~min}$ & $100.0 \%$ & $100.0 \%$ \\
\hline Malaise & $100.0 \%$ & $100.0 \%$ \\
\hline Fever & $15.9 \%$ & $32.8 \%$ \\
\hline Weakness & $100.0 \%$ & $100.0 \%$ \\
\hline Weight Loss & $47.6 \%$ & $65.6 \%$ \\
\hline Muscle Wasting & $31.7 \%$ & $49.2 \%$ \\
\hline $\mathrm{H} / \mathrm{O}$ multiple joint pain and swelling & $100.0 \%$ & $100.0 \%$ \\
\hline $\mathrm{H} / \mathrm{O}$ generalized body ache with sleep disturbances & $100.0 \%$ & $100.0 \%$ \\
\hline Anaemia & $82.5 \%$ & $65.6 \%$ \\
\hline Vasculitis & $4.8 \%$ & $7.4 \%$ \\
\hline Tenosinovitis & $10.7 \%$ & $15.6 \%$ \\
\hline Bursitis & $23.7 \%$ & $29.2 \%$ \\
\hline Reynaud's phenomenon & $11.6 \%$ & $12.8 \%$ \\
\hline Deformities & $17.5 \%$ & $19.2 \%$ \\
\hline
\end{tabular}

Among the study patients morning stiffness, malaise, weakness, h/o multiple joint pain and swelling $\mathrm{h} / \mathrm{o}$ generalized body ache and sleep disturbances were found in $100 \%$ of patients in both groups. Less common manifestations were fever, weight loss, anaemia, vasculitis, tenosinovitis etc found among the study groups. 
Table III: Disease activity parameters in different visits

\begin{tabular}{|c|c|c|c|c|c|c|}
\hline \multirow[t]{2}{*}{ Disease activity } & \multicolumn{2}{|c|}{$\begin{array}{l}\text { Baseline } \\
\text { (Mean ) }\end{array}$} & \multicolumn{2}{|c|}{$\begin{array}{l}\text { 1st visit (10th week) } \\
\text { (Mean) }\end{array}$} & \multicolumn{2}{|c|}{$\begin{array}{l}\text { 2nd visit (14th week) } \\
\text { (Mean) }\end{array}$} \\
\hline & $\begin{array}{c}\text { Group A } \\
(\mathrm{n}=63)\end{array}$ & $\begin{array}{l}\text { Grou } \\
\text { p B }\end{array}$ & Group A $(n=56)$ & $\begin{array}{c}\text { Grou } \\
\mathrm{p} \mathrm{B}\end{array}$ & $\begin{array}{c}\text { Group A } \\
(\mathrm{n}=49)\end{array}$ & $\begin{array}{c}\text { Group } \\
\text { B }\end{array}$ \\
\hline Number of tender joint & 9.21 & 8.98 & 4.90 & 7.02 & 6.37 & 6.68 \\
\hline Number of swollen joint & 7.03 & 8.33 & 2.13 & 3.32 & 1.80 & 1.66 \\
\hline Duration of fatigue in hours & 4.71 & 5.11 & 2.40 & 3.17 & 1.87 & 3.17 \\
\hline VAS [visual analogue score] $(0-10)$ & 7.11 & 7.66 & 4.32 & 6.15 & 4.73 & 5.66 \\
\hline DAS - 28 Score & 6.1741 & 6.0656 & 4.4784 & $\begin{array}{c}5.36 \\
37\end{array}$ & 4.8298 & 5.1020 \\
\hline
\end{tabular}

Among the study patients morning stiffness, malaise, weakness, h/o multiple joint pain and swelling $\mathrm{h} / \mathrm{o}$ generalized bodyache and sleep disturbances were found in $100 \%$ of patients in both groups. Less common manifestations were fever, weight loss, anaemia, vasculitis, tenosinovitis etc found among the study groups.

Table IV: Baseline disease activity among both groups:

\begin{tabular}{|c|c|c|c|c|c|c|c|c|}
\hline \multirow[t]{2}{*}{ Disease activity } & \multirow[t]{2}{*}{ Visit } & \multirow[t]{2}{*}{ Group } & \multirow[t]{2}{*}{$\mathbf{N}$} & \multirow[t]{2}{*}{ Mean } & \multirow{2}{*}{$\begin{array}{c}\text { Std. } \\
\text { Deviation }\end{array}$} & \multirow{2}{*}{$\begin{array}{c}\text { Std. Error } \\
\text { Mean }\end{array}$} & \multicolumn{2}{|c|}{ Significance } \\
\hline & & & & & & & $t$ & $\mathrm{P}$ \\
\hline \multirow[t]{2}{*}{ Number of tender joint } & \multirow[t]{2}{*}{0 week } & Group A & 63 & 9.21 & 2.772 & .349 & \multirow[t]{2}{*}{.591} & \multirow[t]{2}{*}{.556} \\
\hline & & Group B & 61 & 8.98 & 1.008 & .129 & & \\
\hline \multirow[t]{2}{*}{ Number of swollen joint } & \multirow[t]{2}{*}{0 week } & Group A & 63 & 7.03 & 4.068 & .513 & \multirow{2}{*}{2.282} & \multirow[t]{2}{*}{.024} \\
\hline & & Group B & 61 & 8.33 & 1.796 & .230 & & \\
\hline \multirow[t]{2}{*}{ Duration of fatigue in hours } & \multirow[t]{2}{*}{0 week } & Group A & 63 & 4.71 & 3.526 & .444 & \multirow[t]{2}{*}{.714} & \multirow[t]{2}{*}{.477} \\
\hline & & Group B & 61 & 5.11 & 2.640 & .338 & & \\
\hline \multirow{2}{*}{$\begin{array}{c}\text { VAS* } \\
\text { [visual analogue score] }\end{array}$} & \multirow[t]{2}{*}{0 week } & Group A & 63 & 7.11 & 1.357 & .171 & \multirow[t]{2}{*}{2.753} & \multirow[t]{2}{*}{.007} \\
\hline & & Group B & 61 & 7.66 & .750 & .096 & & \\
\hline \multirow[t]{2}{*}{ DAS 28 Score** $^{* *}$} & \multirow[t]{2}{*}{0 week } & Group A & 63 & 6.1741 & .71089 & .08956 & \multirow[t]{2}{*}{.934} & \multirow[t]{2}{*}{.352} \\
\hline & & Group B & 61 & 6.0656 & .57313 & .07338 & & \\
\hline
\end{tabular}

Regarding analysis of different inflammatory parameters during first visit in group A and group B it was found that all parameters were comparable in both groups $(\mathrm{p}>0.05)$. 
Table V: Disease activity among both groups at $2^{\text {nd }}$ visit $\left(10^{\text {th }}\right.$ week $)$

\begin{tabular}{|c|c|c|c|c|c|c|c|c|}
\hline \multirow[t]{2}{*}{ Disease activity } & \multirow[t]{2}{*}{ Visit } & \multirow[t]{2}{*}{ Group } & \multirow[t]{2}{*}{$\mathbf{N}$} & \multirow[t]{2}{*}{ Mean } & \multirow{2}{*}{$\begin{array}{c}\text { Std. } \\
\text { Deviation }\end{array}$} & \multirow{2}{*}{$\begin{array}{l}\text { Std. Error } \\
\text { Mean }\end{array}$} & \multicolumn{2}{|c|}{ Significance } \\
\hline & & & & & & & $\mathrm{t}$ & $\mathrm{P}$ \\
\hline \multirow[t]{2}{*}{ Number of tender joint } & \multirow{2}{*}{$\begin{array}{l}10 \text { th } \\
\text { week }\end{array}$} & Group A & 56 & 4.90 & 4.356 & .553 & \multirow[t]{2}{*}{3.635} & \multirow[t]{2}{*}{.001} \\
\hline & & Group B & 53 & 7.02 & 1.008 & .131 & & \\
\hline \multirow[t]{2}{*}{ Number of swollen joint } & \multirow{2}{*}{$\begin{array}{l}10 \text { th } \\
\text { week }\end{array}$} & Group A & 56 & .00 & .000 & .000 & \multirow[t]{2}{*}{13.656} & \multirow[t]{2}{*}{.001} \\
\hline & & Group B & 53 & 3.32 & 1.916 & .249 & & \\
\hline \multirow{2}{*}{$\begin{array}{c}\text { Duration of fatigue in } \\
\text { hours }\end{array}$} & \multirow{2}{*}{$\begin{array}{l}\text { 10th } \\
\text { week }\end{array}$} & Group A & 56 & 2.40 & 1.541 & .196 & \multirow[t]{2}{*}{2.780} & \multirow[t]{2}{*}{.004} \\
\hline & & Group B & 53 & 3.17 & 1.487 & .194 & & \\
\hline \multirow{2}{*}{$\begin{array}{c}\text { VAS }^{*} \\
\text { [visual analogue score] }\end{array}$} & \multirow{2}{*}{$\begin{array}{l}\text { 10th } \\
\text { week }\end{array}$} & Group A & 56 & 4.32 & .954 & .121 & \multirow[t]{2}{*}{12.035} & \multirow[t]{2}{*}{.001} \\
\hline & & group B & 53 & 6.15 & .690 & .090 & & \\
\hline \multirow[t]{2}{*}{ DAS 28 Score** $^{* *}$} & \multirow{2}{*}{$\begin{array}{l}10 \text { th } \\
\text { week }\end{array}$} & Group A & 56 & 4.4784 & 1.10171 & .13992 & \multirow[t]{2}{*}{5.846} & \multirow[t]{2}{*}{.001} \\
\hline & & Group B & 53 & 5.3637 & .38230 & .04977 & & \\
\hline
\end{tabular}

Regarding analysis of different inflammatory parameters during second visit in group A and group B, statistically significant change of disease activities were found in both groups. $(\mathrm{p}<0.05)$,

Table VI: Disease activity among both groups during $3^{\text {rd }}$ visit $\left(14^{\text {th }}\right.$ week $)$

\begin{tabular}{|c|c|c|c|c|c|c|c|c|c|}
\hline \multirow[t]{2}{*}{ Disease activity } & \multirow[t]{2}{*}{ Visit } & \multirow[t]{2}{*}{ Group } & \multirow[t]{2}{*}{$\mathbf{N}$} & \multirow[t]{2}{*}{ Mean } & \multirow{2}{*}{$\begin{array}{l}\text { Std. } \\
\text { Deviation }\end{array}$} & \multirow{2}{*}{$\begin{array}{l}\text { Std. } \\
\text { Mean }\end{array}$} & \multirow[t]{2}{*}{ Error } & \multicolumn{2}{|c|}{ Significance } \\
\hline & & & & & & & & $\mathbf{t}$ & $\mathbf{P}$ \\
\hline \multirow{2}{*}{$\begin{array}{l}\text { Joint pain/ tenderness } \\
\text { (No of joint) }\end{array}$} & \multirow{2}{*}{14 th week } & Group A & 49 & 6.37 & 3.405 & .440 & & \multirow[t]{2}{*}{0.677} & \multirow[t]{2}{*}{0.50} \\
\hline & & Group B & 47 & 6.68 & .955 & .124 & & & \\
\hline \multirow[t]{2}{*}{ Number of swollen joint } & \multirow[t]{2}{*}{14 th week } & Group A & 49 & 1.80 & 3.052 & .394 & & \multirow[t]{2}{*}{0.286} & \multirow[t]{2}{*}{0.77} \\
\hline & & Group B & 47 & 1.66 & 2.170 & .283 & & & \\
\hline \multirow{2}{*}{$\begin{array}{l}\text { Duration of fatigue in } \\
\text { hours }\end{array}$} & \multirow{2}{*}{ 14th week } & Group A & 49 & 1.87 & 1.096 & .142 & & \multirow[t]{2}{*}{5.44} & \multirow[t]{2}{*}{0.01} \\
\hline & & Group B & 47 & 3.17 & 1.487 & .194 & & & \\
\hline \multirow{2}{*}{$\begin{array}{l}\text { VAS* } \\
\text { [visual analogue score] }\end{array}$} & \multirow[t]{2}{*}{14 th week } & Group A & 49 & 4.73 & 1.351 & .174 & & \multirow[t]{2}{*}{4.61} & \multirow[t]{2}{*}{0.01} \\
\hline & & group B & 47 & 5.66 & .757 & .099 & & & \\
\hline \multirow[t]{2}{*}{ DAS 28 Score $^{* *}$} & \multirow[t]{2}{*}{14 th week } & Group A & 49 & 4.829 & .90822 & .11725 & & \multirow[t]{2}{*}{2.05} & \multirow[t]{2}{*}{0.04} \\
\hline & & Group B & 47 & 5.102 & .46415 & .06043 & & & \\
\hline
\end{tabular}

Regarding analysis of different inflammatory parameters during third visit in group A and group B, statistically significant changes of some disease activities were found in both (duration of fatigue, VAS, DAS28) groups $(p<0.05)$ but not all(joint tenderness, number of swollen joints, $\mathrm{p}>0.05)$. 


\section{Follow up evaluation in Group A (methotraxate and antioxidant) patients:}

Disease activity in relation to joint pain and tenderness, joint swelling, duration of fatigue, VAS and DAS 28 score were found significantly different in follow up at $1^{\text {st }}$ visit (difference between 0 and $10^{\text {th }}$ week) and follow up at $2^{\text {nd }}$ visit (difference between $10^{\text {th }}$ and $14^{\text {th }}$ week). Findings at $1^{\text {st }}$ visit and $2^{\text {nd }}$ visit were found statistically significant $(\mathrm{p}<0.05)$.

Table VIIa: Disease activity (Number of tender joints) in group A

\begin{tabular}{|c|c|c|c|c|c|c|}
\hline Number of tender joints & $\mathbf{N}$ & Mean & Std. Deviation & Std. Error Mean & $\mathbf{F}$ & $\mathbf{p}$ \\
\hline Baseline activity ( 0 week) & 63 & 9.21 & 2.772 & .349 & & \\
\hline 2nd visit (10th week) & 56 & 4.258 & 3.146 & .400 & 10.656 & .001 \\
\hline 3rd visit (14th week) & 49 & 1.433 & 1.522 & .197 & 7.293 & .001 \\
\hline
\end{tabular}

* ANOVA Test

Table VIIb: Disease activity (Joint swelling) in group A

\begin{tabular}{|c|c|c|c|c|c|c|}
\hline Number of swollen joint & N & Mean & Std. Deviation & Std. Error Mean & F & p \\
\hline Baseline activity (0 week) & 63 & 7.03 & 4.068 & .513 & .520 & 13.466 \\
\hline 2nd visit (10th week) & 56 & 7.000 & 4.093 & .001 \\
\hline 3rd visit (14th week) & 49 & 1.800 & 3.052 & .394 & 4.568 & .001 \\
\hline
\end{tabular}

* ANOVA Test

Table VIIc: Disease activity (Duration of fatigue) in group A

\begin{tabular}{|c|c|c|c|c|c|c|}
\hline $\begin{array}{c}\text { Duration of fatigue (hours } \\
\text { /day) }\end{array}$ & $\mathbf{N}$ & Mean & Std. Deviation & Std. Error Mean & F & P \\
\hline Baseline activity (0 week) & 63 & 4.71 & 3.526 & .444 & .257 \\
\hline $\mathbf{2}^{\text {nd }}$ visit (10 & th week) & 56 & 2.194 & 2.023 & .090 & 8.537 \\
\hline $\mathbf{3}^{\text {rd }}$ visit $\mathbf{( 1 4}^{\text {th }}$ week) & 49 & .417 & .696 & 4.637 & .001 \\
\hline
\end{tabular}

* ANOVA Test

Table VIId: Disease activity (Visual analogue scale) in group A

\begin{tabular}{|c|c|c|c|c|c|c|}
\hline Visual analogue scale & $\mathbf{N}$ & Mean & Std. Deviation & Std. Error Mean & $\mathbf{F}$ & $\mathbf{P}$ \\
\hline Baseline activity ( 0 week) & 63 & 7.11 & 1.357 & .171 & & \\
\hline $2^{\text {nd }}$ visit $\left(10^{\text {th }}\right.$ week $)$ & 56 & 2.774 & .895 & .114 & 24.413 & .001 \\
\hline $3^{\text {rd }}$ visit $\left(14^{\text {th }}\right.$ week $)$ & 49 & .400 & .694 & .090 & 4.466 & .001 \\
\hline
\end{tabular}

* ANOVA Test

Table VIIe: Disease activity (DAS 28 score) in group A

\begin{tabular}{|c|c|c|c|c|c|c|}
\hline DAS 28 score & $\mathbf{N}$ & Mean & Std. Deviation & Std. Error & F & P \\
\hline Baseline activity (0 week) & 63 & 6.174 & .710 & .089 & & \\
\hline $\mathbf{2}^{\text {nd }}$ visit $\mathbf{( 1 0}^{\text {th }}$ week) & 53 & 1.70 & .509 & .064 & 26.301 & .001 \\
\hline $\mathbf{3}^{\text {rd }}$ visit $\left(\mathbf{1 4}^{\text {th }}\right.$ week) & 47 & .31 & .388 & .0501 & 6.353 & .001 \\
\hline
\end{tabular}

* ANOVA Test 


\section{Follow up evaluation in Group $B$ (Methotrexate only group) patients:}

Disease activity in relation with number of tender joint, number swollen joint, duration of fatigue in hour, VAS and DAS 28 score were analyzed. Statistically significant change of different parameters were found in follow up at $14^{\text {th }}$ week in all activity markers $(\mathrm{p}>0.05)$ whereas it was found insignificant during follow-up at $10^{\text {th }}$ week in reduction of tender joint, duration of fatigue and DAS 28 score $(\mathrm{p}>0.05)$.

Table VIIIa: Disease activity (number of tender joints) in group B

\begin{tabular}{|c|c|c|c|c|c|}
\hline Number of tender Joint & N & Mean & Std. Deviation & Std. Error Mean & F \\
\hline Baseline activity (0 week) & 61 & 8.98 & 1.008 & .129 & \\
\hline 2nd visit (10th week) & 53 & 4.39 & 2.123 & .310 & 3.223 \\
\hline 3rd visit (14th week) & 47 & 1.33 & 1.023 & .184 & 4.321 \\
\hline
\end{tabular}

Table VIIIb: Disease activity (joint swelling) within group B

\begin{tabular}{|c|c|c|c|c|c|}
\hline Joint swelling (number) & $\mathbf{N}$ & Mean & Std. Deviation & Std. Error Mean & F \\
\hline Baseline activity (0 week) & 61 & 8.33 & 1.796 & .230 & \\
\hline 2nd visit (10th week) & 53 & 6.10 & 5.193 & .410 & 12.122 \\
\hline 3rd visit (14th week) & 47 & 1.321 & 1.052 & .243 & 3.823 \\
\hline
\end{tabular}

* ANOVA Test

Table VIIIc: Disease activity (Duration of fatigue) within group B

\begin{tabular}{|c|c|c|c|c|c|}
\hline $\begin{array}{c}\text { Duration of fatigue(hours } \\
\text { /day) }\end{array}$ & N & Mean & Std. Deviation & Std. Error Mean & F \\
\hline Baseline activity (0 week) & 61 & 5.11 & 2.640 & .338 & .732 \\
\hline 2nd visit (10th week) & 53 & 3.213 & 3.879 & .082 & .087 \\
\hline 3rd visit (14th week) & 47 & .354 & .765 & 3.761 & .001 \\
\hline
\end{tabular}

* ANOVA Test

Table VIIId: Disease activity (Visual analogue scale) within group B

\begin{tabular}{|c|c|c|c|c|c|c|}
\hline Visual analogue scale & $\mathbf{N}$ & Mean & Std. Deviation & Std. Error Mean & $\mathbf{F}$ & $\mathbf{P}$ \\
\hline Baseline activity ( 0 week) & 61 & 7.66 & .750 & .096 & & \\
\hline 2nd visit (10th week) & 53 & 4.987 & .543 & .324 & 22.336 & .001 \\
\hline 3rd visit (14th week) & 47 & .634 & .553 & .230 & 3.554 & .001 \\
\hline
\end{tabular}


Table VIIIe: Disease activity (DAS 28 score) within group B

\begin{tabular}{|c|c|c|c|c|c|}
\hline DAS 28 score & N & Mean & Std. Deviation & Std. Error Mean & F \\
\hline Baseline activity (0 week) & 61 & 6.065 & .573 & .073 & .043 \\
\hline 2nd visit (10th week) & 53 & 2.50 & .412 & .0701 & 3.301 \\
\hline 3rd visit (14th week) & 47 & .42 & .343 & .091 \\
\hline
\end{tabular}

* ANOVA Test

Side effect profile: Regarding side effect analysis hot flush was found common in Group A and other like anemia, oral ulcer, dyspepsia and diarrhea was found in both group A and B.

Table IX: Side effect profile

\begin{tabular}{|l|l|l|l|}
\hline Side effects & $\begin{array}{l}\text { Group A } \\
(\mathbf{n = 6 3 ) ( \% )}\end{array}$ & $\begin{array}{l}\text { Group B } \\
(\mathbf{n = 6 1 ) ( \% )}\end{array}$ & $\begin{array}{l}\text { Total } \\
(\mathbf{n = 1 2 4}) \mathbf{( \% )}\end{array}$ \\
\hline Hot flush & $17(13.7 \%)$ & $2(1.6 \%)$ & $39(31.6 \%)$ \\
\hline Anemia & $6(4.8 \%)$ & $7(5.6 \%)$ & $13(10.4 \%)$ \\
\hline Oral ulcer & $9(7.3 \%)$ & $6(4.6 \%)$ & $15(12.03 \%)$ \\
\hline Dyspepsia & $6(4.8 \%)$ & $2(1.6 \%)$ & $8(17.7 \%)$ \\
\hline Diarrhea & $7(5.6 \%)$ & $2(1.6 \%)$ & $9(7.3 \%)$ \\
\hline Total & $63(50.8 \%)$ & $61(49.2 \%)$ & $124(100.0 \%)$ \\
\hline
\end{tabular}

\section{Discussion}

There has been a great advance in the treatment of patients with RA. Now days many patients are interested in alternative treatment like dietary therapy. Although the etiology of RA is still unknown, the inflammation resulting from the immunological reaction is quite well described. It is known that neutrophil granulocytes, macrophages and lymphocytes are activated, so that reactive oxygen and nitrogen species (RS) are produced. These RS can react with lipid, protein and nucleic acids and are thought to be of importance for the etiology and chronicity of the inflammatory rheumatic diseases [8]. One approach to counteract this oxidative stress situation is the use of antioxidants as therapeutic agents. There is some evidence for a positive effect of antioxidants on clinical symptoms of RA [9]. However, this evidence is weak, and information about the most effective antioxidants, antioxidant doses or combinations are lacking. This intervention study has been tested vitamin E exclusively [10].

In our study, a significant reduction in DAS, pain scale in the form of visual analogue score, number of tender and swollen joints was observed following antioxidant intervention on subsequent visits. Change of the disease activity markers were changed more in $14^{\text {th }}$ than the earlier 0 week and $10^{\text {th }}$ week in term of VAS, duration of fatigue, number of tender joints, number of swollen joints 
and DAS 28 score $(\mathrm{p}<0.05)$. The reduction of DAS 28 was remarkable as all patients had an active (high initial mean DAS of 5.8) longstanding disease and had tried all kinds of disease-modifying anti-rheumatic drugs, including combination therapy. Concentration of antioxidant levels were not measured in our study. This finding were consistent with a study done before where antioxidant reduces the different disease activity score [10].

Regarding analysis of different inflammatory parameters during first visit at 0 week in group A and group B, were found that all parameters were comparable in both groups $(\mathrm{p}>0.05)$. But during $2^{\text {nd }}$ visit at $10^{\text {th }}$ week and $3^{\text {rd }}$ visit at $14^{\text {th }}$ week were found statistically significant regarding changes in disease activities in both groups $(\mathrm{p}<0.05)$. A study done by Richard et al [11] where the potential beneficial effects of an antioxidant intervention on clinical parameters for RA were designed. Eight non-smoking female patients with rheumatoid factor + RA and a Disease Activity Score (DAS 28) higher than 2.5 were enrolled in that study. They found the number of swollen and painful joints were significantly decreased and general health significantly increased, as reflected by a significantly improved (1.6) DAS at $t=10$ weeks. The antioxidant effect was considered beneficial as compared to the scores at 0 week, the DAS significantly reduced after 10 weeks. Increase of the DAS (0.7) after the "wash-out period" at $14^{\text {th }}$ week confirmed a significant relation between changes in clinical condition and antioxidants. So the findings of the present study are consistent with their study.

A limited number of clinical intervention studies are available which support the therapeutic or prophylactic activity of antioxidants in the treatment of RA. These studies were all conducted with an oral intake of $1,200 \mathrm{mg} /$ day of vitamin E. reported that the antioxidant $\alpha$-tocopherol significantly reduced pain parameters in a placebo-controlled double-blind trial following a 3 -week supplementation period. Results from a randomized double-blind parallel group comparison study with a-tocopherol and diclofenac showed that the clinical parameters assessed, e.g. morning stiffness, Ritchie joint index, grip strength and pain, were significantly reduced by vitamin E, with similar effectivity and less side effects as compared to regular drug therapy by diclofenac. Results from other intervention studies have generally been in line with this observations [12].

In our study, the effect of vitamin A, C and $\mathrm{E}$ on the inflammatory response and clinical symptoms were evaluated. In view of the limited number of controlled studies, the supporting evidence for beneficial effects of antioxidants on clinical characteristics of rheumatoid arthritis may be considered limited but promising. Another point is that the potential mechanism of action of antioxidants in rheumatoid arthritis needs further attention. The results indicate there may be potential benefit of using antioxidants Vitamins (A, C and E) in combination with methotrexate in the treatment of RA.

\section{Conclusion}

The use of antioxidant Vitamins (A, C and E) in combination with methotrexate in the treatment of RA is beneficial than that of methotrexate 
alone. To clarify the effective dose of the Antioxidant Vitamins (A, C and E) to get effective result it is needed further study.

\section{Recommendation}

Large, double blind, randomized, placebo-controlled, multi centered trial are needed to establish effect of antioxidant vitamins in combination with methotrexate in the treatment RA.

\section{List of Abbreviations}

ARA: American Rheumatological Association CCP: Cyclical Citrullinated Peptide CRP:C -reactive protein, DAS:Disease activity score, ESR:Erythrocyte sedimentation rate, PAD: Peptidyl Arginine Deaminase, RA: Rheumatoid arthritis, RF:Rheumatoid factor, SJC: Swollen joint count, TJC: Tender joint count VAS :Visual analogue scale

\section{References:}

1. Arnett FC, Edworthy SM, Bloch DA. 2006. The American Rheumatism association. 1987 revised criteria for the classification of rheumatoid arthritis. Arthritis Rheum 31: 315-24.

2. Doherty M, Lanyon P, Ralston SH. 2004. Rheumatoid arthritis. In: Boon NA, Colledge NR, Walker BR, Hunter JAA, editors. Davidson's principle and practice of medicine. $20^{\text {th }}$ ed. London: Churchill Livingstone, $1101-6$.

3. Firestein GS. 2005. Etiology and pathogenesis of rheumatoid arthritis. In: Kelley WN,

4. Melton L. 2006. The antioxidant myth: a medical fairy tale. New Scientist 2006 August

5. Mahajan A, Tandon VR. 2004. Antioxidants and rheumatoid arthritis. J Indian Rheumatol Assoc $12: 139-42$

6. Hitchon CA, El-Gabalawy HS. 2006. Oxidation in rheumatoid arthritis. Arthritis Res Ther 6:26578.

7.Fauci MA. 2011. Approach to articular and musculoskeletal disorder. Harison's Internal Medicine; $18^{\text {th }}$ edi: The Mcgraw- Hill Companies. 325: pp 2000-2020

8.Remans PH, van Oosterhout M, Smeets TJ, Sanders M, Frederiks WM, Reedquist KA et al 2005. Intracellular free radical production in synovial $T$ lymphocytes from patients with rheumatoid arthritis. Arthritis Rheum. 52(7):2003-2009 July.

9. Oliver JE, Silman AJ. 2006. Risk factors for the development of rheumatoid arthritis. Scand J Rheumatol. 35(3):169174

10. Jaswal S, Metha HC, Sood AK, Kaur J. 2003. Antioxidant status in rheumatoid arthritis and role of antioxidant therapy. Clin Chim Acta. 338(1-2):123-129.

11. Richard M, van Vugt, Philip J, Ben A. C. 2008. Antioxidant intervention in rheumatoid arthritis: results of an open pilot study. Clin Rheumatol. 27(6): 771-775.

12. Hagfors L, Leanderson P, Skoldstam L, Anderson J, Johanson G. 2003. Antioxidant intake, plasma antioxidants and oxidative stress in a randomized, controlled, parallel, Mediterranean dietary intervention study on patients with rheumatoid arthritis. Nutr J. 2005; 30:3-9 